\title{
Buenas prácticas en educación patrimonial. Análisis de las conexiones entre emociones, territorio y ciudadanía
}

\author{
José María Cuenca-López, Myriam Martín-Cáceres y Jesús Estepa \\ Universidad de Huelva - España
}

\section{RESUMEN}

Se parte de una perspectiva de la educación patrimonial de carácter interdisiciplinar, simbólica-identitaria, sociocrítica, constructivista y sistémica. El objetivo de la investigación consiste en detectar las buenas prácticas que se desarrollan en los centros educativos para trabajar el patrimonio en conexión con los centros patrimoniales. Se diseñan instrumentos de obtención de información (entrevistas y hojas de registro) y tablas de categorías para el análisis de los datos obtenidos. Con estos instrumentos se analizan las exposiciones y observaciones de experiencias educativas en centros patrimoniales de diversa tipología (históricos, artísticos, etnológicos, científicos y de la naturaleza) de los países que participan en la investigación (España, Portugal, Italia, Argentina, Chile y Estados Unidos). A partir de la información obtenida en este proceso, se determinan criterios básicos de buenas prácticas que permiten el diseño de propuestas educativas, su experimentación y evaluación en las que se interconectan las intervenciones didácticas en centros docentes y patrimoniales. Los resultados evidencian los aspectos clave para desarrollar propuestas de buenas prácticas en educación patrimonial, partiendo de las conexiones entre la conformación de la identidades, la educación emocional y la inteligencia territorial, para abordar la formación de una ciudadanía participativa, crítica y reflexiva en el alumando de enseñanza secundaria obligatoria.

Palabras clave: Educación Patrimonial, Formación ciudadana, Identidad social, Inteligencia territorial, Educación emocional.

\section{Good practices in heritage education. Analysis of connections between emotions, territory and citizenship}

\section{ABSTRACT}

It starts from a perspective of heritage education of an interdisciplinary, symbolic-identity, socio-critical, constructivist and systemic nature. The objective of the research is to detect the good practices that are developed in the educational centers to work the heritage in connection with the heritage centers. Instruments for obtaining information (interviews and registration sheets) and category tables are designed for the analysis of the data obtained. With these instruments, the exhibitions and observations of educational experiences in heritage centers of different types (historical, artistic, ethnological, scientific and nature) of the countries participating in the research (Spain, Portugal, Italy, Argentina, Chile and United States) are analyzed. From the information obtained in this process, basic criteria of good practice are determined. These allow the design of educational proposals, their experimentation and evaluation in which educational interventions are interconnected in educational and heritage centers. The results show the key aspects to develop proposals for good practices in heritage education, based on the connections between identity shaping, emotional education and territorial intelligence, to advocate the formation of a participatory, critical and reflective citizenship in the student of compulsory secondary education.

Key words: Heritage education, citizenship education, social identity, territorial intelligence, emotional education. 


\section{Introducción}

La educación patrimonial se ha convertido en una línea de investigación que ha crecido de manera exponencial en los últimos 20 años, destacando la gran cantidad de tesis doctorales y de publicaciones de alto impacto que se han venido produciendo en los comienzos del siglo XXI (Fontal e Ibáñez, 2017).

La investigación que aquí se presenta pretende analizar las conexiones existentes entre los ámbitos de educación formal y no formal en el marco de la educación secundaria obligatoria. Concretamente el objetivo del estudio consiste en detectar las buenas prácticas que se desarrollan en los centros educativos para trabajar el patrimonio, a partir de actividades en centros patrimoniales (museos y centros de interpretación del patrimonio). Dentro de estas buenas prácticas, el criterio de referencia parte de la consideración de la educación patrimonial como factor que potencia la formación de la ciudadanía en los estudiantes, a través del trabajo con la inteligencia emocional y la inteligencia territorial y partiendo siempre desde una perspectiva interdisciplinar (Cuenca y Estepa, 2017).

Así, a partir de este objetivo general se establecen una serie de objetivos específicos que precisan en mayor medida las intenciones de la investigación:

1. Realizar una actualización teórica sobre la educación patrimonial y su papel en el tratamiento de las inteligencias territorial y emocional, a partir de bibliografía específica y otras fuentes de información.

2. Detectar buenas prácticas en los procesos de enseñanza/ aprendizaje del patrimonio en educación secundaria obligatoria dentro de las relaciones entre instituciones educativas y patrimoniales.

3. Diseñar propuestas didácticas de educación patrimonial acordes con el catálogo de buenas prácticas elaborado, dentro del paradigma didáctico actual, en colaboración con profesionales del campo de la didáctica del patrimonio.

4. Experimentar las propuestas de intervención de educación patrimonial en los centros educativos y patrimoniales abordando la formación de la ciudadanía, mediante el tratamiento de identidades, emociones y del territorio.

El carácter interdisciplinar del estudio, marcado por las conexiones entre las materias que desde una perspectiva holística (Cuenca, 2002) se relacionan con el patrimonio (Ciencias Humanas y Sociales, Ciencias Experimentales y de la Naturaleza,...), se desarrolla de manera internacional en las diferentes fases del estudio tanto en Europa (España, Portugal e Italia) como en América (Argentina, Chile y Estados Unidos), seleccionándose países que cuentan con tradición en estos estudios y que están conformados por sociedades de carácter multicultural, donde las relaciones de identidad juegan un papel crucial.

\section{Marco teórico}

La educación patrimonial tradicionalmente se ha considerado desde la perspectiva del empleo del patrimonio como recurso educativo, para la enseñanza/aprendizaje de la Historia y la Historia del Arte, fundamentalmente, o turístico, en la línea de desarrollar la economía de determinadas zonas con un reconocido potencial patrimonial, prioritariamente también de carácter histórico-artístico.

Durante la década de los 90 del siglo XX empieza a abrirse camino en España un nuevo campo de investigación, orientado al análisis del patrimonio desde una perspectiva educativa (Santacana, 1994), coincidiendo con movimientos similares en el ámbito internacional (Hooper-Greenhill, 1991). Con el cambio de siglo la educación patrimonial sufre un crecimiento exponencial, al reconocerse su potencial didáctico en los ámbitos formales, no formales e informales. Así, empiezan a presentarse resultados de tesis doctorales y proyectos de investigación (Cuenca, 2002; Fontal, 2003; Rennie y Johnston, 2004; Xanthoudaki, Tickle y Sekules, 2003) que reivindican el papel de la educación patrimonial como un elemento clave para el conocimiento e interpretación de las sociedades.

En ese contexto, se presentan diversas actuaciones que abordan el papel social y educativo del patrimonio. En el caso español, se elabora en 2013 el Plan Nacional de Educación y Patrimonio y, en el contexto internacional, destacan los programas desarrollados por la UNESCO y, en especial, la Convention on the Value of Cultural Heritage for Society. A partir de estas propuestas, las nuevas líneas de actuación consideran la necesidad de abordar el patrimonio desde diferentes perspectivas, pero teniendo como base aspectos relacionados con la socialización patrimonial y la formación de una ciudadanía crítica y activa.

El tratamiento educativo de los museos ha sido objeto de estudio desde hace bastantes años, como se puede comprobar en diversas publicaciones (Asensio y Pol, 2001; Domínguez, Estepa y Cuenca, 1999; Estepa, Domínguez y Cuenca, 2001; Estepa, 2013; Hooper-Greenhill, 1991) en las que se analizan el papel educativo de los mismos en relación con los aprendizajes desarrollados por el público en general, y, particularmente, el escolar (Hooper-Greenhill, 2007 y 2013), el impacto de las exposiciones y programas educativos, así como del uso de las tecnologías (Asensio y Asenjo, 2011; Ibáñez, 2011; Ott y Pozzi, 2011; ), culminando con investigaciones mucho más integrales a partir de estudios de caso (Calaf y Suárez, 2015; Martín-Cáceres y Cuenca, 2016) y el empleo de instrumentos de investigación cada vez más afinados (Fontal, García-Ceballos, Arias y Arias, 2019; Martín-Cáceres y Cuenca, 2015).

En el ámbito educativo formal, los estudios se centran fundamentalmente en el análisis de los obstáculos que impiden el desarrollo de propuestas educativas innovadoras, en las que el patrimonio se convierta en el centro de interés. En esta línea, destacan los estudios sobre la formación inicial y la práctica profesional del profesorado, en los que se profundiza sobre los diseños didácticos y las actuaciones de los docentes, tanto en el campo metodológico como en la selección y el tratamiento de los contenidos (Estepa, Ávila y Ferreras, 2008; Maccario, 2012). Junto a las investigaciones centradas en el profesorado, también se ha configurado como línea de estudio el análisis de los materiales didácticos, principalmente libros de texto de Ciencias Sociales, Geografía e Historia, poniendo de relieve los problemas y oportunidades que estos promueven en el tratamiento didáctico del patrimonio (Cuenca y López-Cruz, 2014). Igualmente, el análisis de espacios patrimoniales, como yacimientos arqueológicos, para su tratamiento educativo (Rivero, Fontal, García-Ceballos y Martínez, 2018).

Otro de los principales campos de estudio de la educación patrimonial en el ámbito formal se refiere a los aprendizajes desarrollados por el alumnado y el proceso didáctico, en gran parte relacionado con los materiales producidos en el aula, los contenidos asimilados y la capacidad de relacionarlos entre ellos y con otras materias del curriculum, planteándose como finalidad la formación para una ciudadanía activa, participativa y crítica (Barghi, Zakaria, Hamzah y Hashim, 2017; Okvuran, 2010; Redon, 2019; Simşek, G. y Kesici, 2012; Trabajo y Cuenca, 2017).

En los últimos años, los temas más sobresalientes de investigación en educación patrimonial se han centrado en el análisis de 
las relaciones con la identidad y la formación de la ciudadanía, respecto a los problemas socioambientales relevantes y el tratamiento de las emociones (Cuenca, Estepa y Martín-Cáceres, 2017).

A partir de aquí, se plantea el interés de relacionar la educación emocional (Bisquerra, 2016; Goleman 2008) y la inteligencia territorial (Girardot y Brunau, 2010) con el objeto de promover el desarrollo de la inteligencia ciudadana en la toma de decisiones en un contexto de gobernanza participativa, para tratar las relaciones entre el territorio y la sociedad, considerando el concepto de comunidad territorial (Bertacchini, 2013). De este modo, la educación patrimonial conjuga las relaciones entre emociones y territorio, analizando la conformación de identidades para la formación de una ciudadanía incluyente y participativa (No, Schugurensky y Brennan, 2017), basada en la empatía, el respeto, la convivencia y el bienestar individual y comunitario, así como competente para abordar las tensiones socioecológicas (Miedes, 2019).

Para desarrollar esta perspectiva se han hecho diversas aproximaciones al estudio de las relaciones entre patrimonio, identidad y ciudadanía desde la perspectiva educativa, centrándose en el análisis del territorio (Domínguez- Almansa y López-Facal, 2015), al igual que en de las relaciones entre patrimonio, emociones e identidades (Delgado, 2014; Lucas, 2018), así como los estudios que conectan patrimonio, museos, identidad, ciudadanía y educación (Cuenca, Martín-Cáceres y Schugurensky, 2017; Pinto, 2016; Semedo, 2015), y también aquellos que se centran en la relevancia de su papel en la formación del profesorado (Schugurensky y Wolhuter, 2018).

\section{Método}

El planteamiento de esta investigación se caracteriza por una metodología cualitativa enfocada a partir del interaccionismo simbólico, dentro del paradigma interpretativo/naturalista, en el que se combinan el método etnográfico (Álvarez-Gayou, 2003) y el estudio de caso, a través de la experimentación de diversas propuestas didácticas en diferentes ámbitos socioculturales (Bisquerra, 2009).

Para la consecución de los objetivos de la investigación, indicados en la introducción, se desarrolla una propuesta metodológica que parte de una visión holística en todos los procesos e instrumentos que componen el estudio, abordándose las diferentes fases del estudio desde una perspectiva integral:

1. Realización de entrevistas a gestores patrimoniales y responsables educativos de museos.

2. Observación de experiencias educativas en los centros patrimoniales.

3. Diseño y experimentación de propuestas didácticas elaboradas en centros educativos para su desarrollo en museos.

Se han elaborado y validado diversos instrumentos de investigación para la obtención de datos (entrevistas estandarizadas y en profundidad, diario de campo y observación). Para el análisis de la información se ha empleado una tabla de categorías (tabla 1), caracterizada por el establecimiento de indicadores organizados en función de una hipótesis de progresión sobre la educación patrimonial, que constituye el referente para la interpretación de los resultados del estudio (Cuenca, 2002).

Los informantes de las fases 1 y 2 del estudio consisten en 31 instituciones patrimoniales, prioritariamente museos (tabla 2), seleccionados a partir de diversos criterios: tipología patrimonial, tratamiento social del patrimonio, visión identitaria y de conformación de ciudadanía y el diseño de propuestas en la línea del establecimiento de buenas prácticas en educación patrimonial.
El diseño y experimentación de propuestas didácticas, llevado a cabo en la fase 3, se ha realizado a partir de las observaciones y análisis realizados en las dos primeras fases del estudio, desarrollándose en varios centros educativos de Valparaíso (Chile), Huelva y Cádiz (España).

La información tratada se obtiene de las entrevistas a gestores patrimoniales y docentes, los materiales producidos en el aula durante las experiencias de educación patrimonial desarrolladas y las observaciones de las intervenciones de aula.

\section{Resultados y discusión}

Las fases 1 y 2 del estudio, junto a la revisión bibliográfica sobre experiencias didácticas e investigaciones en educación patrimonial han permitido obtener una visión panorámica de diferentes criterios y actuaciones que permiten determinar buenas prácticas para la enseñanza del patrimonio.

Se han analizado las entrevistas realizadas a los gestores de los museos seleccionados como informantes, así como las observaciones realizadas sobre diversas experiencias educativas en las que se conectan propuestas didácticas realizadas por centros educativos en estos museos.

Para ello se han empleado los instrumentos de toma de datos y de análisis de la información, a través de las tablas de categorías, que guían toda la investigación. Hay diversos museos en los que no se han realizado experiencias didácticas que permitan obtener información sobre buenas prácticas en educación patrimonial: Museo de Artes y Costumbres Populares de Castill de Campos, Conjunto Arqueológico de Carmona, Museo de Écija, Museo Claudio Arrau en Chillán y Museo Pedro del Rio Zañartu, ya que en estas instituciones no se han diseñado propuestas didácticas ni relaciones estables con ningún centro educativo para poder evaluar las conexiones.

Se analizan las actuaciones desarrolladas en el resto de instituciones patrimoniales a partir de las diferentes categorías que marcan el diseño de la investigación. Respecto a la categoría I ¿Para qué enseñar?, se comprueba como, en general, son los museos portugueses los que muestran una mayor aproximación a tener como referente de las propuestas educativas los aspectos relacionados con el análisis del territorio (Museo del Douro en Peso da Rágua, Museo Marítimo de Ílhavo, Museo Regional y Municipal del Algarve), con abundantes conexiones entre museo y escuela. En una línea similar se sitúan el Museo de Historia y Ciencias Naturales de Valparaíso y el Museo de Historia Natural de Concepción, en Chile, o el Parque Nacional de Doñana y el Ecomuseo Molino del Pintado, en España. Todos estos espacios patrimoniales conectan también con propuestas de educación ambiental, en las que las emociones juegan un papel relevante.

Continuando en el ámbito español, tanto en el caso del Museo de la Siderurgia, del Parque de las Ciencias y el Museo del Prado, las propuestas se centran en finalidades vinculadas a la alfabetización científica y/o cultural.

Normalmente, la finalidad perseguida por todas estas instituciones, dentro de las propuestas didácticas que elaboran, se centra en perspectivas academicistas, aunque en algunos pocos casos se observa también una finalidad práctica-conservacionista, situación habitual en las propuestas educativas planteadas en los museos, como se ha puesto en evidencia también en estudios anteriores (Cuenca, Martín-Cáceres, Ibáñez y Fontal, 2014).

Dentro de la categoría II ¿Qué se enseña?, las diferentes instituciones que son objeto de análisis se centran en las tipologías de patrimonio que determinan sus colecciones, aunque normalmente priman las perspectivas temporales y estéticas. Así sucede especialmente en los museos italianos (Museo del patrimonio 
Tabla 1.

Categorías, subcategorías, indicadores y descriptores del estudio (Fuente: elaboración propia).

\section{INDICADORES}

Inteligencia emocional

cional

Alfabetización científica y cultural

1. ¿Hacia qué enfoques se orienta la propuesta?

\section{I. ¿Para qué enseñar?}

\section{II. ¿Qué se enseña?}

Educación ciudadana

Inteligencia territorial

2. ¿Qué finalidad tiene el proceso educativo?

Academicista nalista

Monumental

3. ¿Por qué algo es patrimonio?

Temporal

Simbólico-identitaria
Capacidad del individuo para tomar conciencia de sus emociones, comprender los sentimientos de los demás y desarrollar una actitud empática y social.

Dotar a las personas no sólo de un lenguaje científico, sino enseñarles a desmitificar y decodificar las creencias adheridas a la ciencia y los científicos, prescindir de su aparente neutralidad, entrar en las cuestiones epistemológicas y en las terribles desigualdades ocasionadas por el mal uso de la ciencia y sus condicionantes socio-políticos.

Formar personas política y éticamente activas, conscientes de sus derechos y obligaciones, comprometidas con la defensa de la democracia y los derechos humanos, sensibles y solidarias con las circunstancias de los demás y con el entorno en el que vivimos.

Formar personas conscientes del deterioro del medioambiente y la sobreexplotación Educación Ambiental de los recursos naturales, comprometidas con la defensa y protección de la naturaleza y el desarrollo sostenible.

Capacidad de las personas para tomar conciencia de su territorio como un patrimonio colectivo del que dependen biológica y culturalmente, así como para compartir conocimientos y recursos que permitan construir soluciones colectivas integradas ante los desafíos comunes Conocimiento de hechos e informaciones de carácter cultural, ilustrado y/o centrado en aspectos anecdóticos.

Propagandística Valoración del patrimonio por intereses políticos y/o económicos.

Práctica-conservacio- Valores patrimoniales en la vida cotidiana (económicos, identitarios...) y potencianista ción de su conservación.

Sociocrítica Formación de ciudadanos críticos y comprometidos con la defensa el desarrollo sostenible en el ámbito patrimonial.

Fetichista- Excepcio- Admiración irracional, convirtiéndose en amuleto, objeto de culto o mágico. Escasez, rareza, singularidad y valor crematístico. Grandiosidad y reconocido prestigio, de elementos vivos y no vivos, materiales o inmateriales.

Estética $\quad$ Belleza natural, artística y estilística.
Carácter evolutivo-temporal de los cambios en la naturaleza, especies relictas.

Diversidad Riqueza en bio-geodiversidad y/o diversidad cultural. Elementos simbólicos que caracterizan a una sociedad, un entorno natural, la geo-biodiversidad.

Patr. Natural-HistóriElementos de carácter medioambiental. Referentes arqueológicos y documentales. co-Artístico Manifestaciones correspondientes a los diferentes movimientos estilísticos. Paisajes asociados a ellos.

4. ¿Qué patrimonio se enseña?

Patr. Etnológico

Elementos significativos y tradicionales que explican el cambio social y paisajes asociados a ellos.

Patr. Científico-Tec- Objetos e instrumentos de la ciencia y componentes tecnológicos e industriales de nológico. relevancia social y paisajes asociados.

Patr. Holístico Consideración global e integrada de todas las manifestaciones anteriores.

5. ¿Qué nivel de interrelación existe entre el patrimonio que se enseña?

\section{Unidisciplinar Se trabaja una sola tipología patrimonial.}

Multidisciplinar Se trabajan varios tipos de patrimonio de forma sumativa.

Interdisciplinar Se trabajan varios tipos de patrimonio de forma sistémica. 


CATEGORÍAS SUBCATEGORÍAS INDICADORES DESCRIPTORES

\begin{tabular}{|c|c|c|c|}
\hline \multirow{7}{*}{$\begin{array}{l}\text { II. ¿Qué se } \\
\text { enseña? }\end{array}$} & \multirow{3}{*}{$\begin{array}{l}\text { 6. ¿Cómo se integran } \\
\text { los contenidos? }\end{array}$} & Sin integración & $\begin{array}{l}\text { Se trabaja de forma predominante un tipo de contenidos (Conceptos; Procedimien- } \\
\text { tos; Actitudes). }\end{array}$ \\
\hline & & Integración simple & $\begin{array}{l}\text { Se trabajan de forma predominante dos tipos de contenidos relacionados (C-P; C-A; } \\
\text { P-A). }\end{array}$ \\
\hline & & Integración compleja & Se trabajan los tres tipos de contenidos de forma interrelacionada. \\
\hline & \multirow{4}{*}{$\begin{array}{c}\text { 7. ¿De qué manera } \\
\text { se contextualizan los } \\
\text { contenidos? }\end{array}$} & Funcional & Se presenta el uso y funcionamiento de los elementos patrimoniales. \\
\hline & & Temporal & Se presenta la cronología y el contexto histórico de los elementos patrimoniales. \\
\hline & & Espacial & Se presenta la localización geográfica original de los elementos patrimoniales. \\
\hline & & Social & $\begin{array}{l}\text { Se presentan las características sociales de las comunidades relacionadas con los } \\
\text { elementos patrimoniales tratados. }\end{array}$ \\
\hline \multirow{14}{*}{$\begin{array}{l}\text { III. ¿Cómo se } \\
\text { enseña? }\end{array}$} & \multirow{4}{*}{$\begin{array}{c}\text { 8. ¿Qué presencia } \\
\text { tiene el patrimonio } \\
\text { en el programa edu- } \\
\text { cativo? }\end{array}$} & Presencia anecdótica & $\begin{array}{l}\text { Actividades puntuales y descontextualizadas sin relación con la programación } \\
\text { didáctica. }\end{array}$ \\
\hline & & Recurso didáctico & Fuente de información para el trabajo e interpretación del contexto socioambiental. \\
\hline & & Objetivo específico & $\begin{array}{l}\text { El patrimonio es objeto específico de enseñanza en la propuesta didáctica, empleán- } \\
\text { dose también como recurso. }\end{array}$ \\
\hline & & Contenido educativo & El patrimonio es recurso, objetivo y contenido de la propuesta de enseñanza. \\
\hline & \multirow{3}{*}{$\begin{array}{l}\text { 9. ¿Qué papel juega } \\
\text { el profesorado y } \\
\text { alumnado? }\end{array}$} & $\begin{array}{l}\text { Comunic. Unidirec- } \\
\text { cional }\end{array}$ & Monólogo e inexistencia de interacción. \\
\hline & & $\begin{array}{l}\text { Comunic. Bidirec- } \\
\text { cional }\end{array}$ & Interacción unívoca entre emisor y receptor. \\
\hline & & $\begin{array}{l}\text { Comunic. Multidirec- } \\
\text { cional }\end{array}$ & Interacciones múltiples entre emisores y receptores. \\
\hline & \multirow{3}{*}{$\begin{array}{l}\text { 10. ¿Qué tipo de } \\
\text { actividades se desa- } \\
\text { rrollan? }\end{array}$} & $\begin{array}{l}\text { Complejidad de nivel } \\
\text { cognitivo baja }\end{array}$ & $\begin{array}{l}\text { Se presentan como ejercicios teóricos de carácter descriptivo (buscar datos, copiar y } \\
\text { pegar) basados exclusivamente en la utilización del material curricular. }\end{array}$ \\
\hline & & $\begin{array}{l}\text { Complejidad de nivel } \\
\text { analítico }\end{array}$ & $\begin{array}{l}\text { Se formulan actividades teóricas de índole descriptiva y justificativa que pueden } \\
\text { utilizar el libro de texto u otras fuentes (bibliográficas y telemáticas). }\end{array}$ \\
\hline & & $\begin{array}{l}\text { Complejidad de nivel } \\
\text { sistémico }\end{array}$ & $\begin{array}{l}\text { Se diseñan actividades teórico-prácticas basadas en tareas de descripción, justifica- } \\
\text { ción y argumentación que requieren diversos materiales y fuentes de información. }\end{array}$ \\
\hline & \multirow{4}{*}{$\begin{array}{l}\text { 11. ¿Qué recursos se } \\
\text { emplean? }\end{array}$} & Pasivos tradicionales & Recursos tradicionales que no implican la participación activa del alumnado. \\
\hline & & Pasivos TICs & Recursos TICs que no implican la participación activa del alumnado. \\
\hline & & Activos tradicionales & Recursos que implican la participación activa del alumnado. \\
\hline & & Activos TICs & $\begin{array}{l}\text { Recursos TICs que implican la participación activa del alumnado o público (web } \\
2.0 \ldots \text {... }\end{array}$ \\
\hline \multirow{6}{*}{$\begin{array}{l}\text { IV. ¿Qué re- } \\
\text { laciones se } \\
\text { establecen entre } \\
\text { la inteligencia } \\
\text { emocional y el } \\
\text { patrimonio? }\end{array}$} & \multirow{3}{*}{$\begin{array}{l}\text { 12. ¿Qué dimensio- } \\
\text { nes del aprendizaje } \\
\text { se establecen? }\end{array}$} & Cognitiva & Centrada exclusivamente en el aprendizaje de contenidos e informaciones. \\
\hline & & Emocional & Centra los aprendizajes en las motivaciones y en el interés del alumnado. \\
\hline & & Social & $\begin{array}{l}\text { Centrada en las interacciones con el contexto social dentro de un marco territorial } \\
\text { concreto. }\end{array}$ \\
\hline & \multirow{3}{*}{$\begin{array}{c}\text { 13. ¿Qué habilidades } \\
\text { se desarrollan a tra- } \\
\text { vés de la inteligencia } \\
\text { emocional? }\end{array}$} & $\begin{array}{l}\text { Inteligencia intraper- } \\
\text { sonal }\end{array}$ & $\begin{array}{l}\text { Autoconciencia emocional, asertividad, respeto por uno mismo, auto-actualización e } \\
\text { independencia, manejo del estrés y del estado de ánimo. }\end{array}$ \\
\hline & & $\begin{array}{c}\text { Inteligencia interper- } \\
\text { sonal }\end{array}$ & Empatía, relación interpersonal y responsabilidad social. \\
\hline & & $\begin{array}{l}\text { Adaptación al en- } \\
\text { torno }\end{array}$ & Solución de problemas, contrastación de la realidad y flexibilidad. \\
\hline
\end{tabular}




\begin{tabular}{|c|c|c|c|}
\hline CATEGORÍAS & SUBCATEGORÍAS & INDICADORES & DESCRIPTORES \\
\hline \multirow{4}{*}{$\begin{array}{l}\text { IV. ¿Qué re- } \\
\text { laciones se } \\
\text { establecen entre } \\
\text { la inteligencia } \\
\text { emocional y el } \\
\text { patrimonio? }\end{array}$} & \multirow{4}{*}{$\begin{array}{l}\text { 14. ¿Cuáles son los } \\
\text { elementos constituti- } \\
\text { vos de la identidad/ } \\
\text { patrimonio? }\end{array}$} & Afectivo-emocionales & $\begin{array}{l}\text { Relaciones sociales (familia, amigos, asociaciones) y referentes patrimoniales asocia- } \\
\text { dos a ellas (elementos festivos, gastronomía, útiles de la vida cotidiana). }\end{array}$ \\
\hline & & Territoriales & $\begin{array}{l}\text { Elementos naturales, espaciales y arquitectónicos y paisajes asociados a hechos so- } \\
\text { cio-históricos. }\end{array}$ \\
\hline & & $\begin{array}{l}\text { Convenciones so- } \\
\text { ciopolíticas }\end{array}$ & $\begin{array}{l}\text { Elementos sociopolíticos convencionales (bandera, himno, escudos heráldicos) y to- } \\
\text { dos los referentes patrimoniales tipificados en la legislación. }\end{array}$ \\
\hline & & $\begin{array}{l}\text { Multiplicidad iden- } \\
\text { titaria }\end{array}$ & $\begin{array}{l}\text { Integración de todos los elementos anteriores y valoración y respeto a la diversidad } \\
\text { de identidades. }\end{array}$ \\
\hline \multirow{14}{*}{$\begin{array}{l}\text { V. ¿Qué rela- } \\
\text { ciones se es- } \\
\text { tablecen entre } \\
\text { inteligencia } \\
\text { territorial y } \\
\text { patrimonio? }\end{array}$} & \multirow{3}{*}{$\begin{array}{l}\text { 15. ¿Qué visión del } \\
\text { paisaje se trabaja? }\end{array}$} & Musealizado & Percepción del paisaje como una foto fija, inamovible e inalterable. \\
\hline & & Sostenible & $\begin{array}{l}\text { Percepción de las interacciones del ser humano con la naturaleza y de los proble- } \\
\text { mas asociados a ellas. }\end{array}$ \\
\hline & & Cultural & $\begin{array}{l}\text { Valoración de la identidad y de las percepciones del individuo en relación con la } \\
\text { naturaleza. Comprensión de la naturaleza como parte de la cultura de una sociedad. }\end{array}$ \\
\hline & \multirow{3}{*}{$\begin{array}{l}\text { 16. ¿Qué visión de } \\
\text { la ciudadanía se } \\
\text { trabaja? }\end{array}$} & $\begin{array}{l}\text { Ciudadanía indivi- } \\
\text { dual }\end{array}$ & $\begin{array}{l}\text { Ciudadano personalmente responsable y consciente de sus derechos, con sentido } \\
\text { de pertenencia a una comunidad. Puede participar individualmente en iniciativas } \\
\text { lideradas e impulsadas por otras personas. Reconocimiento del valor simbólico e } \\
\text { identitario exclusivamente de aquellos elementos patrimoniales cercanos y directa- } \\
\text { mente relacionados con el individuo por su experiencia personal. }\end{array}$ \\
\hline & & Ciudadanía social & $\begin{array}{l}\text { Ciudadano participativo y consciente de sus derechos individuales y las respon- } \\
\text { sabilidades con su comunidad. Puede liderar o impulsar el funcionamiento de } \\
\text { organizaciones y/o iniciativas. Reconocimiento del valor simbólico e identitario de } \\
\text { elementos patrimoniales relativos a la cultura propia. }\end{array}$ \\
\hline & & Ciudadanía global & $\begin{array}{l}\text { Ciudadano del mundo y comprometido con la justicia que respeta y valora la inter- } \\
\text { culturalidad. Cuestiona y colabora en la transformación de estructuras y sistemas } \\
\text { que reproducen la injusticia a lo largo del tiempo. Reconocimiento del valor simbó- } \\
\text { lico e identitario de elementos patrimoniales externos a la propia cultura y participa- } \\
\text { ción activa en la gestión del patrimonio. }\end{array}$ \\
\hline & \multirow{4}{*}{$\begin{array}{l}\text { 17. ¿Qué visión del } \\
\text { medioambiente se } \\
\text { trabaja? }\end{array}$} & $\begin{array}{l}\text { Proteccionista-Con- } \\
\text { servacionista }\end{array}$ & $\begin{array}{l}\text { Conservación a ultranza de la naturaleza con soluciones ambientales drásticas que } \\
\text { no tienen en cuenta los efectos, consecuencias o repercusiones socioeconómicas. }\end{array}$ \\
\hline & & Activista & $\begin{array}{l}\text { Perspectiva basada en la implicación para la defensa y protección del medioambien- } \\
\text { te a través de medidas de conservación/protección paliativas. }\end{array}$ \\
\hline & & Desarrollo sostenible & $\begin{array}{l}\text { Consideración de la necesidad de mantener un desarrollo económico a un ritmo que } \\
\text { permita la recuperación o regeneración de los recursos naturales. }\end{array}$ \\
\hline & & Postdesarrollo & $\begin{array}{l}\text { Enfoque basado en propuestas que superan el concepto de desarrollo sostenible. } \\
\text { Consideración de haber alcanzado los límites del desarrollo. }\end{array}$ \\
\hline & \multirow{4}{*}{$\begin{array}{l}\text { 18. ¿Qué conexión } \\
\text { con el entorno se } \\
\text { aborda? }\end{array}$} & Sin conexión & No se establecen conexiones entre patrimonio y entorno. \\
\hline & & Institucional & Se establecen lazos de conexión con las instituciones patrimoniales del entorno. \\
\hline & & Territorial & $\begin{array}{l}\text { Se establecen conexiones entre patrimonio y entorno próximo (barrio, localidad, } \\
\text { Comunidad Autónoma, por ejemplo). }\end{array}$ \\
\hline & & Interterritorial & $\begin{array}{l}\text { Se establecen conexiones entre patrimonio y otros ámbitos territoriales (otras Co- } \\
\text { munidades Autónomas, y regiones). }\end{array}$ \\
\hline
\end{tabular}

industriale, Museo Nazionale e biblioteca della Musica, Museo Tamo y Museo della Citta di Rimini), con propuestas didácticas unidisciplinares y fundamentalmente de carácter histórico-artístico. Situación similar ocurre en muchos museos chilenos ( $\mathrm{Mu}$ seo Baburizza) y españoles (Museo del Prado). Un enfoque más holístico con un tratamiento interdisciplinar del patrimonio se presenta en el Ecomuseo Molino del Pintado, aunque centrado, de una u otra manera, en el patrimonio histórico y etnológico.

Los museos norteamericanos perciben el término patrimonio desde una visión diferente a la concepción europea. A pesar de que se mantiene una visión temporal y estética, centrada en el patrimonio histórico-artístico (especialmente en los grandes museos nacionales), tambien hay interesantes visiones sociales y participativas, desde la perspectiva de los museos comunitarios, como sucede en los casos de Arizona Science Center, Heard Museum o Tempe History Museum, por ejemplo.

La diferencia más evidente se presenta en los museos portugueses, donde los criterios que prevalecen en las exposiciones se centran en la diversidad cultural y perspectivas simbólico-identitarias y holísticas, predominando la presentación del patrimonio etnológico. 
Tabla 2.

Centros patrimoniales participantes en el estudio

\begin{tabular}{|c|c|}
\hline INSTITUCIÓN & PAÍS \\
\hline Heard Museum (Phoenix, Arizona) & \multirow{3}{*}{ Estados Unidos } \\
\hline Tempe History Museum (Tempe, Arizona) & \\
\hline Arizona Science Center (Phoenix, Arizona) & \\
\hline $\begin{array}{l}\text { Ecomuseo Molino Mareal El Pintado (Ayamon- } \\
\text { te, Huelva) }\end{array}$ & \multirow{9}{*}{ España } \\
\hline Conjunto Arqueológico de Carmona (Sevilla) & \\
\hline Museo Histórico Municipal de Écija (Sevilla) & \\
\hline $\begin{array}{l}\text { Museo de artes y costumbres populares de } \\
\text { Castill de Campos (Priego de Córdoba) }\end{array}$ & \\
\hline Museo Nacional de El Prado (Madrid) & \\
\hline $\begin{array}{l}\text { Parque Nacional de Doñana (Huelva, Sevilla, } \\
\text { Cádiz) }\end{array}$ & \\
\hline Museo de la Siderurgia (Asturias) & \\
\hline Museo Municipal de Calatayud (Zaragoza) & \\
\hline Parque de las Ciencias (Granada) & \\
\hline Museu do Douro (Peso da Régua) & \multirow{6}{*}{ Portugal } \\
\hline Museu de Penafiel (Penafiel) & \\
\hline Museu Marítimo de Ílhavo (Ílhavo) & \\
\hline Museu Regional do Algarve (Faro) & \\
\hline Museu Municipal (Faro) & \\
\hline Museu do Traje (Sao Bras de Alportel) & \\
\hline Museo della Citta (Rimini) & \multirow{4}{*}{ Italia } \\
\hline Museo Tamo (Ravenna) & \\
\hline $\begin{array}{l}\text { Museo Nezionale e Biblioteca della Musica } \\
\text { (Bologna) }\end{array}$ & \\
\hline Museo del Patrimonio Industriale (Bologna) & \\
\hline Museo del Limarí La Serena (Ovalle) & \multirow{7}{*}{ Chile } \\
\hline Museo Claudio Arrau (Chillán) & \\
\hline Museo de Historia Natural (Concepción) & \\
\hline $\begin{array}{l}\text { Parque-Museo Pedro del Río Zañartu (Concep- } \\
\text { ción) }\end{array}$ & \\
\hline $\begin{array}{l}\text { Museo de la Historia y Ciencias Naturales (Val- } \\
\text { paraíso) }\end{array}$ & \\
\hline Palacio de Baburizza (Valparaíso) & \\
\hline Museo de Rioja (Valparaíso) & \\
\hline Museo de la Memoria (Rosario) & \multirow{2}{*}{ Argentina } \\
\hline Centro Ana Frank (Buenos Aires) & \\
\hline
\end{tabular}

La contextualización de los contenidos de la mayor parte de los museos analizados es temporal y espacial antes que funcional y solo algunos museos chilenos y portugueses, el P. N. de Doñana, Calatayud o el Tempe History Museum presentan características sociales de las comunidades relacionadas con los elementos patrimoniales tratados.

Respecto a la categoría III ¿Cómo se enseña?, las propuestas didácticas radican en las conexiones entre escuela y museo, para detectar las necesidades educativas necesarias para el tratamien- to del patrimonio por parte del alumnado, como sucede de forma sistemática en el Museo do Traje de Saõ Bras de Alportel y en el Museo Municipal de Calatayud, aunque en otros casos las propuestas de actividades parten más del museo que del centro educativo, como hemos podido observar en otro estudio anterior (Toharia, Gordillo y Pelegero, 2013; Sánchez, 2013), así sucede en el Parque de las Ciencias o en el Museo del Prado.

Las propuestas más interesantes se basan en el fomento de la interacción estudiante-patrimonio a través de dinámicas de comunicación multidireccional y la realización de actividades de nivel analítico y sistémico. Para ello, se diseñan talleres didácticos, visitas dinamizadas y dialogadas a través del análisis de problemáticas culturales, sociales o mediambientales, en la línea del diseño de las VTS llevadas a cabo en los Museos Picasso de Málaga y Barcelona (González-Sanz, Feliú y Cardona, 2017). También es muy interesante el desarrollo de propuestas de investigación en el aula en las que el museo es una fuente relevante de información y donde los resultados pueden ser objeto de presentación, como una exposición del mismo museo. Sin embargo, en muchas ocasiones se pone de manifiesto una concepción del patrimonio academicista, excepcionalista y unidisciplinar, como se puede comprobar en otros estudios (Jiménez, Cuenca y Ferreras, 2010), alejado del potencial que el uso de la tecnología podría significar para trabajar desde una perspectiva más motivadora e interactiva (Cabero y Barroso, 2018).

La categoría IV se centra en cuestionar las relaciones que se establecen entre inteligencia emocional y patrimonio en las propuestas didácticas de los museos y su tratamiento por parte de los centros educativos. En este sentido, hay que poner en evidencia que son pocas las instituciones patrimoniales que abordan este aspecto. Sin embargo, se pueden destacar las propuestas didácticas del Tempe History Museum, que, como ejemplo de los museos comunitarios, aborda un tratamiento social del patrimonio, atendiendo a la multiplicidad identitaria y a la adaptación al entorno. Las observaciones realizadas en el Heard Museum también se vinculan a aspectos emocionales, a pesar de ser un museo de diferentes características, pero su temática relacionada con los problemas de la inmigración norteamericana, convierte a las emociones en uno de los ejes centrales del discurso museístico (Cuenca, Molina y Martín-Cáceres, 2018).

Por supuesto, se presenta un gran acercamiento a la educación vinculada al patrimonio en los museos argentinos de La Memoria y de Ana Frank, en los que además se plantea un tratamiento del conflicto como eje estructurador de los diseños comunicativos y educativos de estas instituciones.

También se pueden percibir algunas visiones incipientes dirigidas a la educación emocional en experiencias desarrolladas en el Museo del Prado y en el Parque Nacional de Doñana

Finalmente, la categoría $\mathrm{V}$ analiza las relaciones que se establecen entre inteligencia territorial y patrimonio. En este caso, los espacios patrimoniales vinculados al medioambiente y a la naturaleza como el Parque Nacional de Doñana, el Museo de Historia Natural y Parque-Museo Pedro del Río Zañartu o el Museo de la Historia y Ciencias Naturales, tienen entre sus premisas el análisis del territorio, incorporando el factor humano y el análisis de su impacto en mayor o menor medida.

Los centros portugueses, de carácter más sociohistórico, perciben un mayor interés por contextualizar sus propuestas en el marco del análisis del territorio desde perspectivas complejas. Así, se encuentran experiencias y propuestas que conectan con el conocimiento social del territorio en museos como el Museo del Douro, el Museo Marítimo de Ílhavo, el Museo Regional y Municipal del Algarve. 
La tercera fase consiste en el diseño y experimentación de propuestas didácticas en diferentes centros educativos, partiendo de la colaboración con diferentes instituciones patrimoniales. Para el diseño de estas propuestas se ha partido de la información obtenida en la observación de las diferentes exposiciones y experiencias didácticas realizadas en los museos, que permitieron la determinación de unos criterios básicos de buenas prácticas que se ponen en evaluación en estas experiencias llevadas a cabo en la tercera fase.

Estas experiencias han tenido lugar en un centro de Valparaíso (Chile), en una unidad didáctica de Historia, Geografía y Ciencias Sociales para abordar las conexiones de la escuela con el museo de Historia Natural de la ciudad de Valparaíso. En España, se realizan dos experimentaciones, en Isla Cristina (Huelva) y en San Fernando (Cádiz). En ambos casos se trata de unidades didácticas cuyo contenido habitualmente no conecta con el patrimonio como, "Los sectores económicos", trabajando en el Ecomuseo Molino Mareal El Pintado (Ayamonte) y "La Materia", en el Molino de Mareas de San Fernando, dentro de la asignatura de Física y Química.

Los resultados obtenidos en las tres experiencias han sido bastante satisfactorios, considerándose el patrimonio como eje estructurador para abordar el tratamiento educativo de las emociones, de las identidades y de la comprensión del territorio, para la formación de los estudiantes en una ciudadanía crítica, participativa y constructiva.

La mayoría de los obstáculos no han venido tanto por la selección de unos contenidos tradicionalmente poco relacionados con el patrimonio (la materia o la economía), ya que los docentes y los estudiantes supieron ver las conexiones existentes y la posibilidad de concebir el patrimonio como un núcleo aglutinador y motivador, sino por las concepciones de los gestores y educadores de los museos y por las propias exposiciones y materiales producidos por estas instituciones, que no percibieron el potencial del patrimonio para trabajar el territorio desde una perspectiva holística y social (más fácil y asequible fue el tratamiento de las emociones y las identidades), con el objetivo de formar ciudadanos comprometidos socialmente.

\section{Conclusiones}

El estudio desarrollado ha permitido detectar diversas propuestas y actividades didácticas que se pueden evaluar como buenas prácticas en el ámbito de la educación patrimonial. Teniendo nuestro sistema de categorías como referencia, se consideran buenas prácticas las que parten de un tratamiento holístico del concepto de patrimonio y una visión integrada del mismo, con actividades didácticas que atienden al componente emocional de la educación patrimonial y al sentido social y comunitario del análisis territorial, siendo siempre las identidades un factor clave, para que se pueda formar al alumnado en una ciudadanoía comprometida, activa y crítica.

Todo ello ha permitido la elaboración de un catálogo de buenas prácticas, a partir del cual se han diseñado varias propuestas didácticas, que se han experimentado y evaluado, para valorar el potencial de las conexiones

El proceso de investigación ha puesto de manifiesto la necesidad de profundizar en las relaciones entre los centros educativos y los centros patrimoniales, de manera que el éxito de cualquier propuesta didáctica depende en gran medida de la coordinación existente entre ambos contextos educativos, formales y no formales. Las experiencias didácticas en este sentido deben convertirse en ejemplos de un proceso de simbiosis, en el que uno no tiene sentido sin el otro, para desarrollar proyectos de educación patrimonial innovadores y efectivos.

En primer lugar, es fundamental tener en cuenta que el fin del proceso educativo es la formación ciudadana de los estudiantes, para ello, el patrimonio y el museo se convierten en un medio, no en el fin. Esto implica una visión social del sentido de la educación patrimonial, en el que se aborden globalmente la identidad cultural, el territorio y las emociones de manera participativa.

Esta finalidad educativa se puede conseguir siempre que los contenidos patrimoniales se conecten con otros contenidos socialmente relevantes, que pueden ser contextualizados, representados e interpretados mediante el uso del patrimonio como recurso. Así, es necesario partir de la visión holística, en la que la integración en las propuestas didáctcas de todas las tipologías patrimoniales permite un mejor y más complejo acercamiento a la realidad sociocultural y medioambiental.

En el plano de la metodología didáctica, las propuestas más exitosas parten del fomento de la interacción de los estudiantes, entre ellos y con el patrimonio, así como con los docentes y mediadores culturales-patrimoniales. No se puede dudar de la potencialidad de la investigación en el aula, mediante los ABP, analizando problemas socialmente relevantes desde perspectivas particiativas, en el que el centro de atención no es el contenido, sino el alumnado. Para ello, lo deseable sería el uso de actividades y recursos activos, ya sean tradicionales (talleres, representaciones,...) o mediente el uso de las TICs (webs 3.0, foros, chats, videojuegos,...), que de una manera $u$ otra fomenten la comunicación multidireccional, la participación y la dinamicidad de las tareas.

Finalmente, una vez que las experimentaciones desarroladas han constatado esta realidad con respecto a las buenas prácticas en el campo de la educación patrimonial, en la línea de la formación de una ciudadanía activa, reflexiva y comprometida con el cambio y progreso social, hay que tener en cuenta un aspecto clave, la formación del profesorado para que estos profesionales de la educación estén adecuadamente capacitados para llevar a cabo el diseño y experimentación de materiales y propuestas didácticas en este sentido.

\section{Referencias bibliográficas}

Álvarez-Gallou, J. L. (2003). Cómo hacer investigación cualitativa. Fundamentos y metodología. México D.C.: Paidós Educador.

Asensio, M., y Asenjo, E. (2011). Lazos de luz azul: museos y tecnologías 1, 2 y 3.0. Barcelona: UOC.

Asensio, M., y Pol, E. (2001). Nuevos escenarios en educación: aprendizaje informal sobre el patrimonio, los museos y la ciudad. Buenos Aires: Aique.

Ávila, R. (2001). Historia del arte, enseñanza y profesores. Sevilla: Diada.

Barghi, R., Zakaria, Z., Hamzah, A. \& Hashim, N. H. (2017). Heritage Education in the Primary School Standard Curriculum of Malaysia. Teaching and Teacher Education, 61, 124-131. DOI: 10.1016/j.tate.2016.10.012

Bertachini, Y. (2013). Entre información y procesos de comunicación La inteligencia territorial. De Prácticas y Discursos: Cuadernos de Ciencias Sociales, 2.

Bisquerra, R. (2009). Metodología de la Investigación educativa. Madrid: La Muralla.

Bisquerra, R. (2016). Educación emocional: 10 ideas clave. Barceolona: Graó.

Cabero, J. y Barroso, J. (2018). Los escenarios tecnológicos en Realidad Aumentada (RA): posibilidades educativas. 
Aula Abierta, 47 (3), 327-336. https://doi.org/10.17811/rifie.47.3.2018.327-336

Calaf, R., y Suárez, M. A. (2015). Acción educativa en museos. Su calidad desde la evaluación cualitativa. Gijón: Trea.

Cuenca, J. M. (2002). El patrimonio en la Didáctica de las Ciencias Sociales: concepciones, dificultades y obstáculos para su integración en la enseñanza obligatoria. Tesis doctoral. Universidad de Huelva.

Cuenca, J. M., Estepa, J., y Martín-Cáceres, M. J. (2017). Patrimonio, educación, identidad y ciudadanía. Profesorado y libros de texto en la enseñanza obligatoria. Revista de Educación, 375, 136-159. https://doi.org/10.4438/1988-592XRE-2016-375-338

Cuenca, J. M., y Estepa, J. (2017). Educación patrimonial para la inteligencia territorial y emocional de la ciudadanía. Midas, 8, 1-11. DOI: $10.4000 /$ midas. 1173

Cuenca, J. M., y López-Cruz, I. (2014). La enseñanza del patrimonio en los libros de texto de Ciencias Sociales, Geografía e Historia para ESO. Cultura y Educación, 26 (1), 19-37. https:/ / doi.org/10.1080/11356405.2014.908663

Cuenca, J. M., Martín-Cáceres, M., y Schugurensky, D. (2017). Educación para la ciudadanía e identidad en los museos de Estados Unidos: análisis desde la perspectiva de la educación patrimonial. Estudios Pedagógicos, 43 (4), 29-48. https://doi. org/10.4067/s0718-07052017000400002

Cuenca, J. M., Martín-Cáceres, M., Ibáñez, A., y Fontal, O. (2014). La educación patrimonial en las instituciones patrimoniales españolas. Clío: History and History Teaching, 40, 1-8. http:/ / clio.rediris.es/n40/articulos/mono/MonCuencaetal2014.pdf

Cuenca, J. M., Molina, S., y Martín-Cáceres, M. (2018). Identidad, ciudadanía y patrimonio. análisis comparativo de su tratamiento didáctico en museos de estados unidos y España. Arbor, 194 (788) http://arbor.revistas.csic.es/index.php/arbor/article/view/2260/3180

Delgado, E. (2014). Educación para la ciudadanía en la enseñanza de las ciencias sociales y su vinculación con las dimensiones de la memoria: estudio de caso en ESO. Tesis doctoral. Universidad de Huelva. http://rabida.uhu.es/dspace/ handle/10272/8841

Domínguez, C., Estepa, J., y Cuenca, J. M. (1999). El museo: un espacio para el aprendizaje. Huelva: Universidad de Huelva.

Domínguez-Almansa, A., y López-Facal, R. (2015). Patrimonio, entorno y procesos de identificación en la educación primaria. Clío: History and History Teaching, 41, 1-20. http:/ / clio. rediris.es/n41/articulos/mono/MonFacal2015.pdf

Estepa, J. (2013) (ed.). La educación patrimonial en la escuela y el museo: investigación y experiencias. Huelva: Universidad de Huelva.

Estepa, J., Ávila, R., y Ferreras, M. (2008). Primary and Secondary Teachers' Conceptions about Heritage and Heritage Education: a Comparative Analysis. Teaching and Teacher Education, 24(8), 2095-2107. DOI: 10.1016/j.tate.2008.02.017

Estepa, J., Domínguez, C., y Cuenca, J. M. (2001). Museo y Patrimonio en la Didáctica de las Ciencias Sociales. Huelva: Universidad de Huelva.

Fontal, O. (2003). La educación patrimonial. Teoría y práctica para el aula, el museo e Internet. Gijón: Trea.

Fontal, O., y Ibáñez, A. (2017) La investigación en educación patrimonial. Evolución y estado actual a través del análisis de indicadores de alto impacto. Revista de Educación, 375, 184214. DOI: $10.4438 / 1988-592 X-R E-2016-375-340$

Fontal, O., García-Ceballos, S., Arias, B., y Arias, V. (2019). Assessing the Quality of Heritage Education Programs: Construction and Calibration of the Q-Edutage Scale. Revista de
Psicodidáctica, 24 (1), 31-38. https://doi.org/10.1016/j.psicoe.2018.11.001

Girardot, J., y Brunau, E. (2010). Inteligencia territorial e innovación para el desarrollo socio-ecológica transición. En 9th International conference of territorial intelligence, ENTI.

Goleman, D. (2008). Inteligencia emocional. Barcelona: Kairós.

González-Sanz, M., Feliú, M., y Cardona, G. (2017). Las Visual Thinking Strategies (VTS) desde la perspectiva del educador patrimonial. DAFO del método en su aplicación práctica. $R e-$ vista de Educación, 375, 160-183. DOI: 10.4438/1988-592X-RE2016-375-339

Hooper-Greenhill, E. (1991). Museum and Gallery Education. Leicester: Leicester University Press.

Hooper-Greenhill, E. (2007). Museum and education. Purpose, pedagogy, performance. London - New York: Routledge.

Hooper-Greenhill, E. (2013). Museums and their visitors. London: Routledge.

Ibáñez, A. (2011). Museums, social media \& 2.0 technology. Bilbao: Universidad del País Vasco.

Jiménez, R., Cuenca, J. M., y Ferreras, M. (2010). Heritage education: Exploring the conceptions of teachers and administrators from the perspective of experimental and social science teaching. Teaching and Teacher Education, 26(6), 1319-1331. DOI: $10.1016 /$ j.tate.2010.01.005.

Lucas, L. (2018). La enseñanza del patrimonio y de la ciudadanía en las clases de ciencias sociales. Un estudio de caso. Universidad de Huelva. Tesis doctoral.

Maccario, N. K. (2012). Stimulation of Multiple Intelligence by Museum Education at Teachers' Training. Procedia - Social and Behavioral Sciences, 51, 807-811.

Martín-Cáceres, M., y Cuenca, J. M. (2016). Communicating heritage in museums: outlook, strategies and challenges through a SWOT analysis. Museum Management and Curatorship, 31 (3), 299-316.

Martín-Cáceres, M. y Cuenca, J. M. (2015). Educomunicación del patrimonio. Educatio Siglo XXI, 33(1), 33-54.

Miedes, B. (2019). Cross-fertilizing roads between Heritage and Participatory Citizenship. Huelva: Faro Convention in Research-Action.

No, W., Schugurensky, D., y Brennan, A. (2017). By the People: Participatory Democracy, Civic Engagement and Citizenship Education. Phoenix: ASU.

Okvuran, A. (2010). The relationship between arts education, museum education and drama education in elementary education. Procedia - Social and Behavioral Sciences, 2 (2), 53895392. https://doi.org/10.1016/j.sbspro.2010.03.878

Ott, M. \& Pozzi, F. (2011). Towards a new era for Cultural Heritage Education: Discussing the role of ICT. Computers in $\mathrm{Hu}$ man Behavior, 27 (4), 1365-1371. https://doi.org/10.1016/j. chb.2010.07.031

Pinto, H. (2016). Educação Histórica e Patrimonial: conceções de alunos e professores sobre o passado em espaços do presente. Porto: CITCEM

Redon, S. (2019). Ciudadanía y participación en redes sociales: un estudio en adolescentes chilenos. Aula Abierta, 48 (2), 203212. https:/ / doi.org/10.17811/rifie.48.2.2019.203-212

Rennie, L. J. \& Johnston, D.J. (2004). The nature of learning and its implications for research on learning from museums. Science Education, 88, (S1), S4-S16.

Rivero, P., Fontal, O., García-Ceballos, S., y Martínez, M. (2018). A model for heritage education through archaeological sites: The case of roman city of Bilbilis. Curator, the museum journal, 61(2), 315-326. 
Sanchéz, B. (2013). El Prado en familia. En J. Estepa (Ed.). La educación patrimonial en la escuela y el museo: investigación y experiencias (pp. 325-334). Huelva: Universidad de Huelva.

Santacana, J. (1994). Didáctica del patrimonio arqueológico: el Proyecto del Poblado Ibérico de Alorda Park o Les Toixoneres de Calafell. Tesis doctoral. Universidad de Valladolid.

Schugurensky, D., y Wolhuter, C. (2018). Global Citizenship Education in Teacher Education:Rheoretical and Practical Issues. Taylor \& Francis

Semedo, A. (2015). Representações e identidade em exposições de museus. Clío. History and History Teaching, 41, 1-26. http:// clio.rediris.es/n41/articulos/mono/MonAsemedo2015.pdf

Sim凶ek, G., y Kesici, A. E. (2012). Heritage Education for Primary School Children Through Drama: The Case of Aydın, Turkey.
Procedia - Social and Behavioral Sciences, 46, 3817-3824. https:// doi.org/10.1016/j.sbspro.2012.06.153

Toharia, M., Gordillo, J. A., Pelegero, V. (2013). La función del patrimonio en los museos de ciencia interactivos. En J. Estepa, J. (Ed.). La educación patrimonial en la escuela y el museo: investigación y experiencias (pp. 335-342). Huelva: Universidad de Huelva.

Trabajo, M., y Cuenca, J. M. (2017) La educación patrimonial para la adquisición de competencias emocionales y territoriales del alumnado de secundaria. Pulso, 40, 159-174.

Xanthoudaki, M., Tickle, L. y Sekules, V. (2003). Researching visual arts education in museums and galleries. An international reader. Dortrecht-Boston-London: Kluwer Academic Publishers. 